\title{
An Iterative Phase Autofocus Approach for ISAR Imaging of Maneuvering Targets
}

\author{
Binbin Wang ${ }^{1}{ }^{\circledR}$, Hao Cha ${ }^{1}$, Zibo Zhou ${ }^{2}$, Huatao Tang ${ }^{1}$, Lidong Sun ${ }^{1}$, Baozhou Du ${ }^{3}$ and Lei Zuo ${ }^{1, *}$ \\ 1 College of Electronic Engineering, Naval University of Engineering, Wuhan 430033, China; \\ bbwang2016@126.com (B.W.); hchanue@163.com (H.C.); tanghuatao2008@126.com (H.T.); \\ donggeaixiao7@163.com (L.S.) \\ 2 Air Force Early Warning Academy, Wuhan 430019, China; zibo_travel@163.com \\ 3 No. 63850 Unit of PLA, Baicheng 137001, China; zhangdongxiaooec@sina.com \\ * Correspondence: leizuonue@163.com
}

check for updates

Citation: Wang, B.; Cha, H.; Zhou, Z.; Tang, H.; Sun, L.; Du, B.; Zuo, L. An Iterative Phase Autofocus Approach for ISAR Imaging of Maneuvering Targets. Electronics 2021, 10, 2100. https://doi.org/10.3390/ electronics10172100

Academic Editor: Byung Cheol Song

Received: 22 July 2021

Accepted: 24 August 2021

Published: 30 August 2021

Publisher's Note: MDPI stays neutral with regard to jurisdictional claims in published maps and institutional affiliations.

Copyright: (c) 2021 by the authors. Licensee MDPI, Basel, Switzerland. This article is an open access article distributed under the terms and conditions of the Creative Commons Attribution (CC BY) license (https:// creativecommons.org/licenses/by/ $4.0 /)$.

\begin{abstract}
Translational motion compensation and azimuth compression are two essential processes in inverse synthetic aperture radar (ISAR) imaging. The anterior process recovers coherence between pulses, during which the phase autofocus algorithm is usually used. For ISAR imaging of maneuvering targets, conventional phase autofocus methods cannot effectively eliminate the phase error due to the adverse influence of the quadratic phase terms caused by the target's maneuvering motion, which leads to the blurring of ISAR images. To address this problem, an iterative phase autofocus approach for ISAR imaging of maneuvering targets is proposed in this paper. Considering the coupling between translational phase errors and quadratic phase terms, minimum entropy-based autofocus (MEA) method and adaptive modified Fourier transform (MFT) are performed iteratively to realize better imaging results. In this way, both the translational phase error and quadratic phase terms induced by target's maneuvering motion can be compensated effectively, and the globally optimal ISAR image is obtained. Comparison ISAR imaging results indicates that the new approach achieves stable and better ISAR image under a simple procedure. Experimental results show that the image entropy of the proposed approach is 0.2 smaller than the MEA method, which validates the effectiveness of the new approach.
\end{abstract}

Keywords: inverse synthetic aperture radar (ISAR); maneuvering targets imaging; iterative phase autofocus

\section{Introduction}

Inverse synthetic aperture radar plays an important role in civil and military fields [1-4]. It can generate the range-Doppler images to describe the size, shape, and structure of the targets. The obtaining of a range-Doppler image relies on the target's motion, which can be decomposed into two parts: translational motion and rotational motion. Translational motion causes incoherence between pulses and has to be compensated, and the rotational motion contributes to the azimuth imaging of the ISAR image.

As for ISAR imaging of maneuvering targets, the existing methods [3,5,6] always assume that the translational motion compensation has been accomplished and focus on the azimuth compression. However, translational motion compensation is an essential step and needs to be considered carefully. For translational motion compensation of maneuvering targets, it is found that conventional range alignment algorithms are still effective. while the translational phase autofocus algorithms need to be considered carefully [7]. Conventional translational phase autofocus algorithms can be divided into two categories: parameterbased approaches and non-parameter-based approaches.

The core idea of the parameter-based approach is resuming the trajectory of the target, which is usually modeled as a polynomial. Furthermore, the optimal polynomial 
coefficients are then obtained through optimization methods such as particle swarm optimization [8]. Finally, translational phase compensation is completed by the translational motion component reconstructed from the modeled trajectory. The parameter-based approach can effectively eliminate the phase error if the translational motion is precisely modeled. However, this approach can only be applied in intermediate frequency direct sampling data [9] and is not suitable for commonly used stretch echo data [2].

The non-parameter-based approach includes Doppler centroid tracking (DCT) [10], phase gradient autofocus (PGA) [11,12], and minimum entropy-based autofocus (MEA) [13,14]. DCT assumes that the rotation-induced phase differences between adjacent pulses can be ignored, and the incoherent phase of two adjacent pulses is calculated by employing the conjugate product of two pulses. However, DCT ignores target's rotational motion, and the compensation results need to be improved. PGA can estimate the gradient of the phase error in ISAR images and obtain the phase error among adjacent pulses by iteration. However, the iteration number is usually uncertain and often leads to a high computational burden. MEA belongs to optimization methods and is founded on the fact that the optimal image corresponds to minimum image entropy. This method is widelyused in practical ISAR imaging radar for its stable convergence, fine imaging results and reasonable computational cost. However, MEA assumes that the target's motion is smooth, which is not satisfied for the ISAR imaging of maneuvering targets. Consequently, the residual phase compensation errors adversely affect the subsequent azimuth compression and significantly degrade the image quality.

In our previous work [15], the method for azimuth compression of ISAR imaging of maneuvering targets was investigated. Specifically, the relative chirp rate (RCR) is defined to describe the target's motion status, and a modified Fourier transform (MFT) is constructed for azimuth compression. Furthermore, an efficient initialization process is designed for the gradient descent method, and the high-quality imaging results of maneuvering targets can be achieved adaptively. Experimental results validated the effectiveness of the method. This study extends the work in [15] by considering both translational phase compensation and azimuth compression. Considering the coupling between translational phase errors and quadratic phase terms caused by the target's maneuvering motion, MEA and adaptive MFT are carried out iteratively to realize better imaging results. Ultimately, the translational phase error and quadratic phase terms can be eliminated effectively, and a well-focused ISAR image is achieved. The significant contributions of this study are summarized as follows: solution for translational phase compensation for ISAR imaging of maneuvering targets; combination of translational phase compensation and azimuth compression to obtain a global optimal ISAR image; unification of the imaging of maneuvering targets and smooth moving targets into the same model and a well-focused image can be obtained adaptively.

The remainder of this paper is organized as follows. In Section 2, the imaging signal model of maneuvering targets is described, Section 3 elaborates the proposed approach based on iterative autofocus, and Section 4 validates the new imaging approach through experiments, followed by conclusions in Section 5 .

\section{ISAR Imaging Signal Model of Maneuvering Targets}

The typical geometry of the ISAR imaging is shown in Figure 1. XOY defines a Cartesian coordinate system with target rotating center at the origin $O$. The radar is located on the target plane along $Y$-axis. Assuming that $P\left(x_{i}, y_{i}\right)$ represents a scatterer on the target, its range to the radar $R_{i}\left(t_{m}\right)$ can be approximated as

$$
R_{i}\left(t_{m}\right)=r\left(t_{m}\right)+x_{i} \sin \theta\left(t_{m}\right)+y_{i} \cos \theta\left(t_{m}\right)
$$

where $r\left(t_{m}\right)$ is the instantaneous distance between radar and the target center, $t_{m}$ is the slow time, and $\theta\left(t_{m}\right)$ is the rotational angle relative to the line of sight. 
Usually, the rotational angel is small during the coherent processing interval, and thus the trigonometric functions in Equation (1) can be approximated as

$$
\begin{aligned}
& \sin \theta\left(t_{m}\right) \approx \theta\left(t_{m}\right) \\
& \cos \theta\left(t_{m}\right) \approx 1
\end{aligned}
$$

Furthermore, then $R_{i}\left(t_{m}\right)$ turns into

$$
R_{i}\left(t_{m}\right)=r\left(t_{m}\right)+x_{i} \theta\left(t_{m}\right)+y_{i}
$$

Suppose that the linear frequency modulated signal is adopted as the transmitted signal, which can be expressed as

$$
s\left(\hat{t}, t_{m}\right)=\operatorname{rect}\left(\frac{\hat{t}}{T_{p}}\right) \exp \left\{j 2 \pi\left(f_{c} t+\frac{1}{2} \gamma \hat{t}^{2}\right)\right\},
$$

where $\operatorname{rect}(\cdot)$ stands for the unit rectangular function, $\hat{t}$ is the fast time, $T_{p}$ denotes the pulse duration, $f_{c}$ is the carrier frequency, and $\gamma$ is the frequency modulation rate.

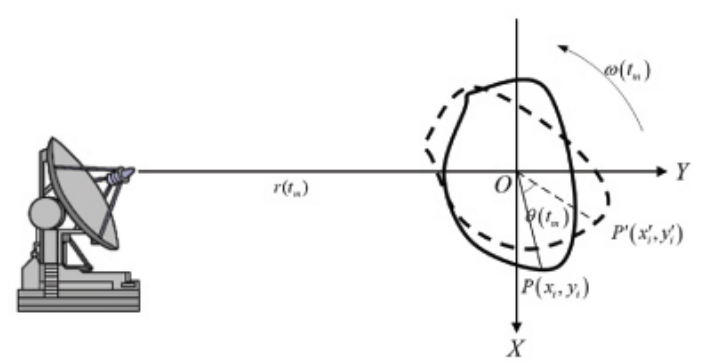

Figure 1. Geometry of the ISAR imaging system.

Assuming that the target model contains $Q$ scatterer points, and $\sigma_{i}$ is the backward scattering coefficient of the $i$-th scatterer point. After pulse compression, the radar echoes can be expressed as

$$
s_{i f}\left(f_{i}, t_{m}\right)=\sum_{i=1}^{Q} \sigma_{i} T_{p} \sin c T_{p}\left(f_{i}+2 \gamma \frac{R_{i}\left(t_{m}\right)}{c}\right) \cdot \exp \left[-j \frac{4 \pi f_{c}}{c} R_{i}\left(t_{m}\right)\right]
$$

where $c$ is light velocity.

For targets with a smooth motion, $\theta\left(t_{m}\right)$ can be described by the uniform rotation model and $\theta\left(t_{m}\right)=\omega t_{m}$, where $\omega$ is the equivalent angular velocity. Then $R_{i}\left(t_{m}\right)$ can be written as

$$
R_{i}\left(t_{m}\right)=r\left(t_{m}\right)+x_{i} \omega t_{m}+y_{i}
$$

In the process of azimuth compression, for simplicity, only the phase part of Equation (5) is considered. Consequently, substituting Equation (6) into (5) yields:

$$
s_{i f}\left(f_{i}, t_{m}\right)=\sum_{i=1}^{Q} \sigma_{i} T_{p} \exp \left[-j \frac{4 \pi f_{c}}{c} r\left(t_{m}\right)\right] \cdot \exp \left[-j \frac{4 \pi f_{c}}{c}\left(x_{i} \omega t_{m}+y_{i}\right)\right]
$$

The first phase term in (7) is the phase error induced by the translational motion, and the second one corresponds to the range and azimuth positions of scatterers.

After compensating the phase error, the ISAR image can be obtained by performing Fourier transform along the azimuth direction. Fourier transform is the conventional azimuth compression algorithm in ISAR imaging. We denote the aligned range profile sequence as $G(m, n)$, where $m$ is the pulse number related to slow time, and $n$ represents the range bin related to fast time. The total number of pulses and range bins are $M$ and 
$N$, respectively. We denote the discrete ISAR image as $I(m, n)$, and the Fourier transform matrix as $P$. The compensation matrix for the phase error is

$$
\tilde{\boldsymbol{\Theta}}=\left[\begin{array}{ccc}
\Delta \theta_{1} & 0 & 0 \\
0 & \ddots & 0 \\
0 & 0 & \Delta \theta_{m}
\end{array}\right]
$$

The process of RD (abbreviation for range-Doppler) imaging can be expressed as

$$
I=P \cdot \tilde{\Theta} \cdot G
$$

However, when the motion of the target is maneuvering, uniformly accelerated rotation model is applied to describe $\theta\left(t_{m}\right)$. Herein, the rotational angle can be expressed as $\theta\left(t_{m}\right)=\omega t_{m}+\frac{1}{2} \alpha t_{m}^{2}$, where $\alpha$ is the angular acceleration. Furthermore, $R_{i}\left(t_{m}\right)$ becomes

$$
R_{i}\left(t_{m}\right)=r\left(t_{m}\right)+x_{i}\left(\omega t_{m}+\frac{1}{2} \alpha t_{m}^{2}\right)+y_{i}
$$

In this case, Equation (7) turns into:

$$
s_{i f}\left(f_{i}, t_{m}\right)=\sum_{i=1}^{Q} \sigma_{i} T_{p} \exp \left[-j \frac{4 \pi f_{c}}{c} r\left(t_{m}\right)\right] \exp \left[-j \frac{4 \pi f_{c}}{c}\left(x_{i} \omega t_{m}+\frac{1}{2} x_{i} \alpha t_{m}^{2}+y_{i}\right)\right]
$$

Inspecting Equation (11) reveals that the translational phase errors exp $\left[-j \frac{4 \pi f_{c}}{c} r\left(t_{m}\right)\right]$ and the quadratic phase terms $\exp \left[-j \frac{4 \pi f_{c}}{c}\left(\frac{1}{2} x_{i} \alpha t_{m}^{2}\right)\right]$ induced by target's maneuvering motion will lead to the burring of the ISAR image. Only when both of them are well compensated can the well-focused ISAR image be obtained. Next, we concentrate on the compensation of phase error and quadratic phase term in the following section.

\section{Imaging Approach Based on Iterative Phase Autofocus}

\subsection{Algorithm Discription}

To settle the quadratic phase terms induced by the maneuvering of the targets, our previous work proposed an adaptive imaging method based on an MFT, in which a new variable called relative chirp rate (RCR) is defined to construct and then compensate the quadratic phase terms in the MFT matrix. The definition of MFT and estimation of RCR has been discussed in detail in [15], so only a brief description will be provided here to avoid duplication. The definition of RCR is

$$
K_{\alpha \omega}=\frac{2 x_{i} \alpha / \lambda}{2 x_{i} \omega / \lambda}=\frac{\alpha}{\omega}
$$

It is worth mentioning that the RCR is identical for all scatterers on the target, and the value of the RCR can be used as an indication of the target's maneuvering. When the RCR is large, it means that the equivalent angular acceleration is large and the maneuvering of the target is severe. Thus, the quadratic phase terms are relatively large. In contrast, a lower maneuvering target corresponds to a small RCR.

The construction of MFT matrix containing quadratic phase compensation is summarized as following. If we define $W=\exp \left(-j \frac{2 \pi}{M}\right)$, an element in the conventional Fourier transform matrix can be expressed as

$$
P\left(m, m^{\prime}\right)=W^{m^{\prime} m}
$$


where $m$ and $m^{\prime}$ are the index of the row and column, respectively. In like manner, the element in quadratic phase compensation matrix $P_{c}$ can be written as

$$
P_{c}\left(m, m^{\prime}\right)=W^{-m^{\prime} m^{2} \frac{K_{\alpha \omega} T}{2\left(1+0.5 K_{\alpha \omega} M T\right)}}
$$

where $T$ is the pulse repeat interval. The MFT can be expressed as

$$
P^{\prime}\left(K_{\alpha \omega}\right)=P \odot P_{c}
$$

where $\odot$ is the Hadamard product. The imaging process of maneuvering targets can be expressed as

$$
I=P^{\prime}\left(K_{\alpha \omega}\right) \cdot \tilde{\Theta} \cdot G
$$

It should be noticed that the RCR is zero for targets with smooth motion, and MFT degrades to Fourier transform in this instance.

Equation (16) indicates that the mathematical principle in ISAR imaging of maneuvering targets differs from that for smooth moving targets, as expressed in Equation (9). However, the commonly used iterative processes in the MEA method are deduced based on Equation (9). When the MEA method is applied to the phase autofocus of maneuvering targets, Equation (9) cannot be satisfied, and the blurring appears in the ISAR images. It can be inferred that the blurring comes from the residual translational compensation errors.

To compensate the residual translational compensation errors, we have defined a variable called Doppler focus shift (DFS) in our previous work [15]. In simple terms, DFS is the Doppler cell numbers between Doppler center and Doppler focus, where the Doppler center is defined as the Doppler cell in which the azimuth coordinate is zero, and the Doppler focus is the Doppler cell in which the equivalent rotation axis located. In general, they are located in the same Doppler cell. When the translational motion compensation error exists, they may not coincide. Visual representation of DFS is shown in Figure 2.

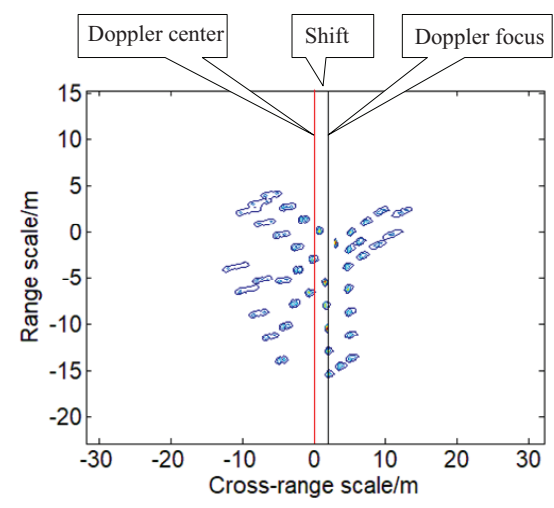

Figure 2. Representation of Doppler focus shift.

It should be noted that the DFS is derived from the residual linear translational compensation error, which we defined as the linear phase error. In an ISAR image, each Doppler cell is related to a row vector in Fourier transform. Thus, the effect of DFS on ISAR imaging can be eliminated by circle shift of the row vector in the adaptive MFT process. However, when the residual translational compensation errors are non-linear, they cannot be compensated by DFS.

To further eliminate the residual translational phase error of maneuvering targets, an iterative phase autofocus approach is proposed in this paper. Considering that the phase error $\Theta_{m}$ and $P^{\prime}\left(K_{\alpha \omega}\right)$ are coupled with each other, the new approach alternately updates the $\tilde{\Theta}_{m}$ and $P^{\prime}\left(K_{\alpha \omega}\right)$ so that they can both converge to the optimal solution. Through the adaptive MFT, the ISAR image blurring caused by the quadratic phase terms is reduced significantly. Then the inverse Fourier transform (IFT) is performed along the azimuth direction of ISAR image, and the updated range profiles are obtained. In the update of 
range profiles, the maneuvering degree of target is alleviated. Next, the $\tilde{\Theta}_{m}$ and $P^{\prime}\left(K_{\alpha \omega}\right)$ are estimated again. The combination of IFT and adaptive MFT reduces the maneuvering of the target at the signal level. Through iterations, the quadratic phase terms induced by the target's maneuvering motion are compensated and the value of RCR becomes very small. When the value of the RCR is smaller than a threshold $K_{\alpha \omega}^{t h}$, we believe that the maneuvering of the target is compensated effectively. In this paper, we set the threshold of the RCR to $K_{\alpha \omega}^{t h}=10^{-4}$. After that, the equivalent accelerated rotation of the maneuvering target was converted to uniform, and the assumption in Equation (9) was satisfied. The phase error $\Theta$ can be precisely estimated by MEA. By now, both of the quadratic phase terms and translational phase error were well compensated.

Detailed implementation procedures of the iterative phase autofocus approach for maneuvering targets are given as follows:

Step 1 Complete the pulse compression and range alignment of radar echoes;

Step 2 Obtain the estimation of $\tilde{\Theta}_{m}$ through MEA algorithm;

Step 3 Accomplish the azimuth compression by adaptive MFT, and then the intermediate ISAR image is obtained and denote the corresponding RCR is $K_{\alpha \omega}$;

Step 4 Check if $K_{\alpha \omega}<K_{\alpha \omega}^{t h}$. If it is, the intermediate ISAR image is the optimal image; if not, perform IFT along the azimuth of the intermediate ISAR image and go to Step 2.

To summarize, the flowchart of new imaging approach is illustrated in Figure 3.

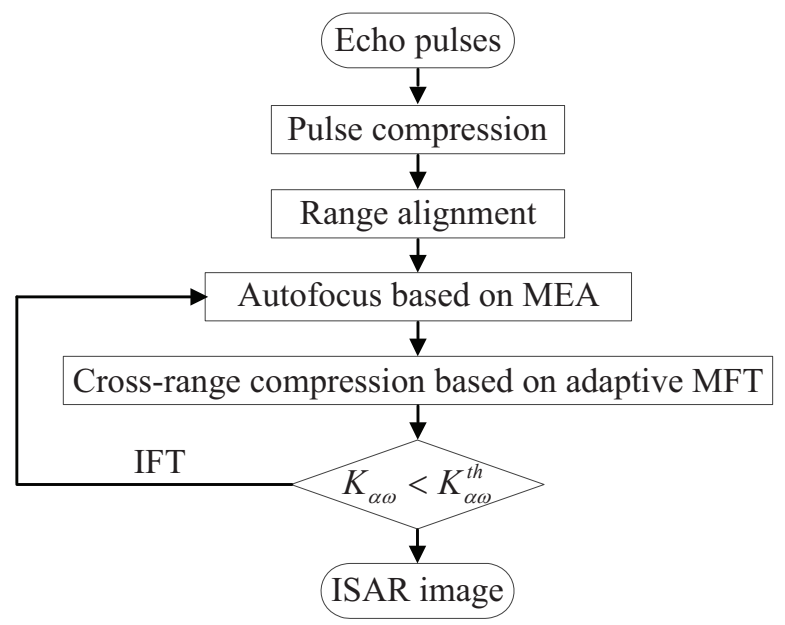

Figure 3. Flowchart of the new imaging approach.

\subsection{Algorithm Discussion}

In general, phase autofocus and azimuth compression for ISAR imaging of maneuvering targets are two separate processes. In ISAR imaging of maneuvering, after phase autofocus, range instantaneous Doppler methods or parameterestimation-based methods are always applied to reconstruct ISAR images. The result of phase autofocus will directly affect the final imaging quality. However, in the new approach, the two processes have been unified. The phase errors caused by translational motion and the quadratic phase terms induced by target's maneuvering motion have been estimated alternately. As a result, both of them converge to the optimal solution, and the global optimal image is achieved.

Furthermore, the new imaging approach is also suitable for smooth-moving targets. After range compression and translational motion compensation, a high-quality image can be obtained by performing Fourier transform along the azimuth direction. The above is the main process of the conventional RD imaging algorithm.

When the new approach is applied for ISAR imaging of smooth moving target, as is shown in Figure 3, after translational motion compensation, adaptive MFT is used to accomplish azimuth compression as a substitution of Fourier transform. Due to the 
motion of the target being smooth, the equivalent angular acceleration during the coherent processing interval is zero, and hence, RCR is zero. In this moment, MFT degrades to Fourier transform. The value of RCR is smaller than the preset threshold, and the iterative process is naturally terminated. Furthermore, the new imaging approach degrades to conventional RD algorithm.

From the former analysis, it can be seen that the new approach unifies the translational phase autofocus of maneuvering targets and smooth-moving targets into the same model. Moreover, it can adaptively obtain a global optimal image, whether the motion of the targets is maneuvering or smooth. This is very useful and convenient for practical ISAR imaging systems.

\section{Simulation Results}

In this section, simulated airplane data and Boeing B727 data are utilized to verify the effectiveness of the proposed iterative phase autofocus approach. The imaging results of the new imaging approach are compared with those obtained by (1) RD algorithm, (2) adaptive MFT algorithm, and (3) adaptive MTF with DFS compensation, in terms of image quality.

\subsection{Imaging Results of an Airplane Model}

In the simulation, the assumed target is an airplane model composing 42 isotropic scatterers, as shown in Figure 4a. The parameter settings of the radar system are illustrated in Table 1 . The target's coordinates in the radar coordinate system are $(3000,3000$, $7000) \mathrm{m}$ and the target's speed is $v=(225,300,0) \mathrm{m} / \mathrm{s}$. We set target's acceleration as $a=(84,112,0) \mathrm{m} / \mathrm{s}^{2}$. Modified envelope correlation [16] and MEA are utilized for range alignment and translational phase autofocus, respectively.

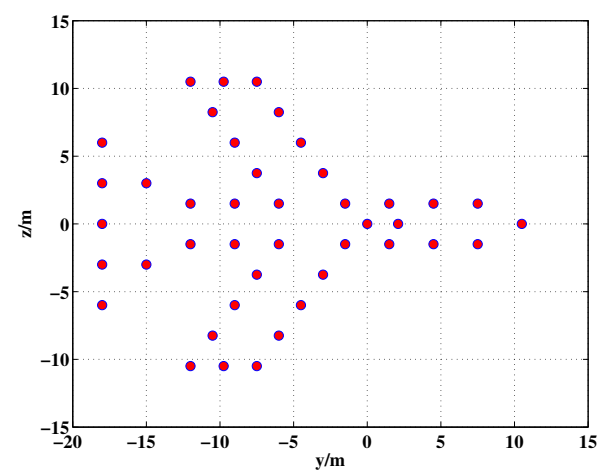

(a)

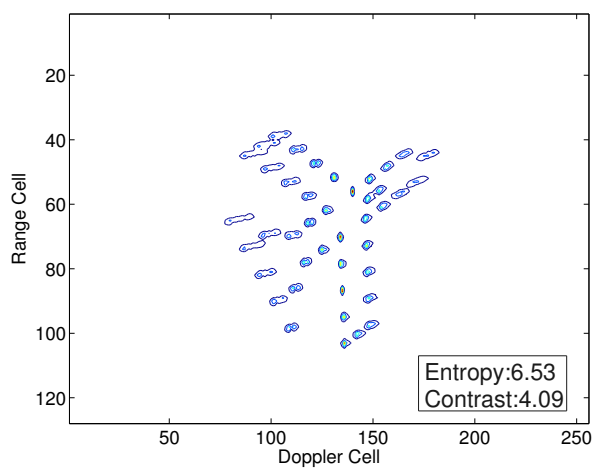

(b)

Figure 4. Target model and RD image. (a) Target model. (b) ISAR image based on RD algorithm.

Table 1. Parameters of the radar system.

\begin{tabular}{lclc}
\hline Parameter & Setting Value & Parameter & Setting Value \\
\hline Carrier frequency & $10 \mathrm{GHz}$ & Bandwidth & $500 \mathrm{MHz}$ \\
Pulse width & $50 \mu \mathrm{s}$ & Complex sampling rate & $500 \mathrm{MHz}$ \\
Pulse repeat frequency & $200 \mathrm{~Hz}$ & Pulse number & 256 \\
\hline
\end{tabular}

As shown in Figure $4 b$, a focused image cannot be obtained by conventional RD algorithm due to the acceleration of the target. Image entropy and contrast [17] are applied 
to quantitatively evaluate the imaging quality. The mathematical definitions of image entropy and contrast are given in Equations (17) and (18), respectively.

$$
\begin{aligned}
& H=-\sum_{m=1}^{M} \sum_{n=1}^{N} p(m, n) \cdot \ln \{p(m, n)\} \\
& \text { where } p(m, n)=\frac{|I(m, n)|}{\sum_{m=1}^{M} \sum_{n=1}^{N}|I(m, n)|} \\
& C=\frac{\sqrt{E\left\{[|I(m, n)|-E(|I(m, n)|)]^{2}\right\}}}{E(|I(m, n)|)}
\end{aligned}
$$

where $E(\cdot)$ represents the average operation. The smaller entropy and higher contrast indicate better-focused ISAR image. It can be seen from Figure $4 \mathrm{~b}$ that image entropy and contrast of the RD image are 6.53 and 4.09 , respectively.

In the image obtained by adaptive MFT, shown in Figure 5a, Doppler resolution has been improved significantly compared with that of the RD, as indicated by the smaller entropy and larger contrast. However, the scatterers in the red ellipse are still slightly blurred, which is caused by the phase distortion problem of MEA and the residual quadratic phase terms. The imaging result requires further improvement in image focal quality. Now, we take DFS into consideration, and the imaging result is provided in Figure 5b. The running result indicates that the optimal RCR and DFS are 0.28 and -9.99 , respectively. It can be seen that the blurring problem in the red ellipse has been effectively alleviated and a high-quality ISAR image is obtained. A smaller entropy and larger contrast also indicates that a higher image quality is achieved.

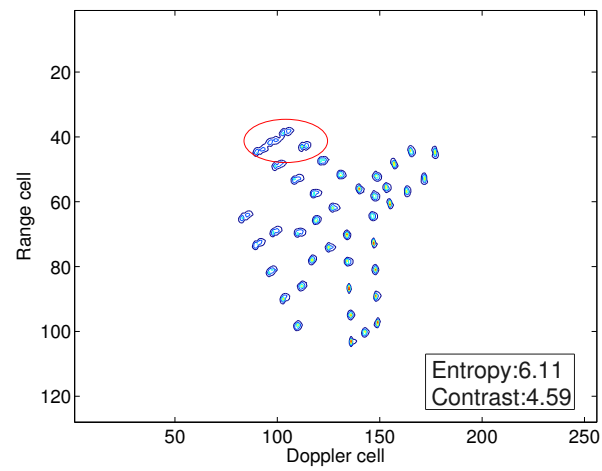

(a)

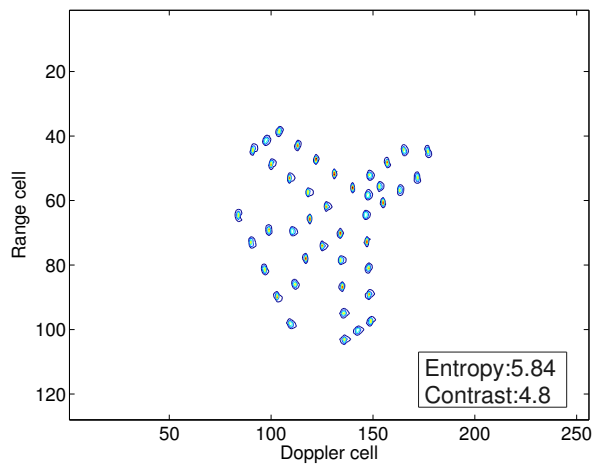

(b)

Figure 5. ISAR images of an airplane model. (a) ISAR image based on adaptive MFT. (b) ISAR image based on adaptive MFT with DFS compensation.

Next, the proposed approach is implemented to obtain the imaging result. To highlight the effectiveness of the new imaging approach, Figure 6a presents the variation of the image entropy during the iterations. The initial value is set as the entropy of the RD image. It can be noticed from Figure $6 a$ that the image entropy decreased gradually. After 3 iterations, the termination condition is met. In the first iteration, the corresponding value of RCR is 0.21 and the image entropy is 6.11. In the following two iterations, the values of RCR are 0.06 and 0 , respectively. Figure $6 \mathrm{~b}$ shows the optimal image based on the new approach, in which the scatterers are well focused in both range and cross-range directions. Compared with the images obtained by other three methods, the image entropy of Figure $6 \mathrm{~b}$ is the smallest, and the contrast is the largest. Therefore, the image focal quality obtained by the new approach is the best, in terms of quantitative image quality evaluations of both entropy and contrast. 


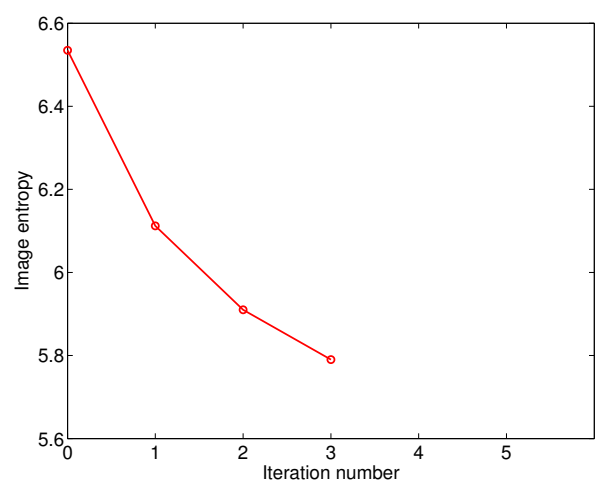

(a)

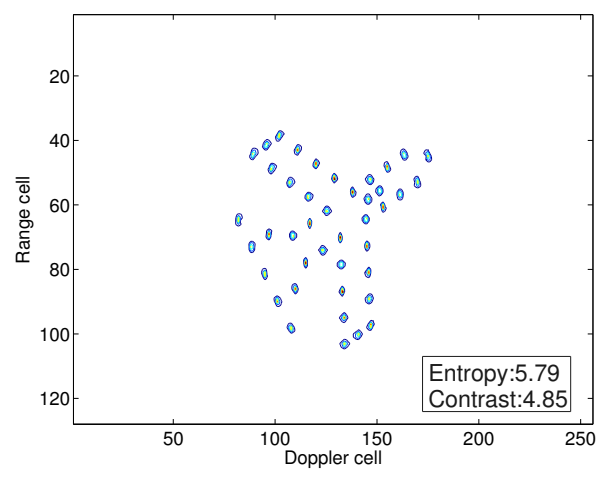

(b)

Figure 6. Experimental results using the new imaging approach. (a) Variation of the image entropy. (b) ISAR image based on the new imaging approach.

The performance of traditional RD, MFT, and MFT with DFS compensation and the proposed approach under different noise levels was analyzed. The data were obtained from the original data by adding Gaussian white noise with different SNRs ranging from $-5 \mathrm{~dB}$ to $5 \mathrm{~dB}$. One hundred Monte Carlo trials were performed, and the average image entropy was collected under different SNR in Figure 7. It can be observed that the image entropy of the proposed algorithm is smallest under each SNR, which further validates the effectiveness of the newly proposed approach.

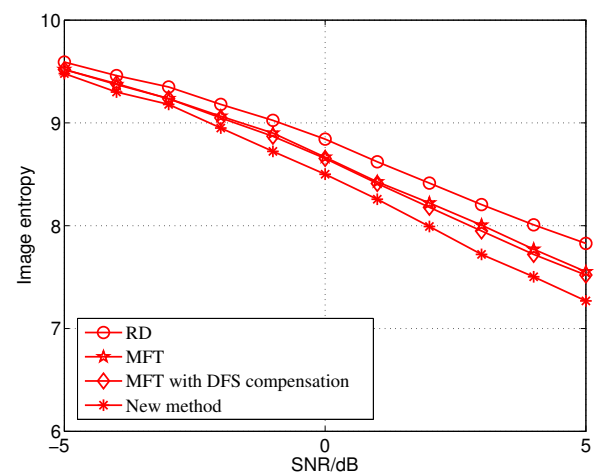

Figure 7. Variation of average image entropy with SNR by using different methods.

\subsection{Imaging Results of Boeing B727}

THe Boeing B727 data set provided by the U.S. Naval Research Laboratory [18] was utilized to verify the effectiveness of the new approach. The carrier frequency and bandwidth of the transmitted signal were $9 \mathrm{GHz}$ and $150 \mathrm{MHz}$, respectively. The pulse repeat frequency was $20 \mathrm{KHz}$. Translational motion compensation was accomplished. Two hundred fifty-six pulses were coherently processed for ISAR image formation, as shown in Figure 8a. The image obtained by RD algorithm is shown in Figure 8b, from which it can be observed that the image quality is quite poor. The image is significantly spread along the Doppler direction owing to the maneuvering of the target. Image entropy and contrast of the RD image are 6.64 and 2.4 , respectively. 


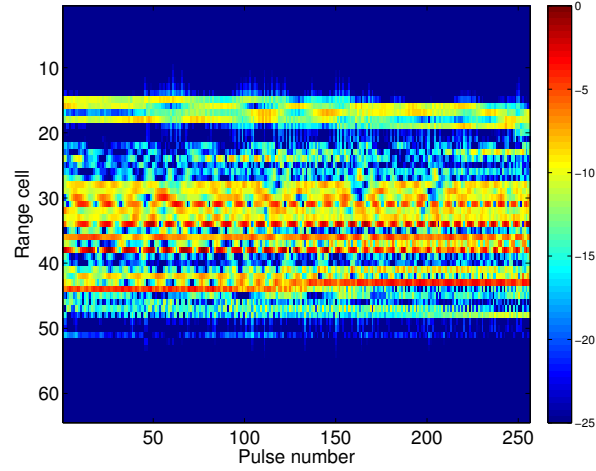

(a)

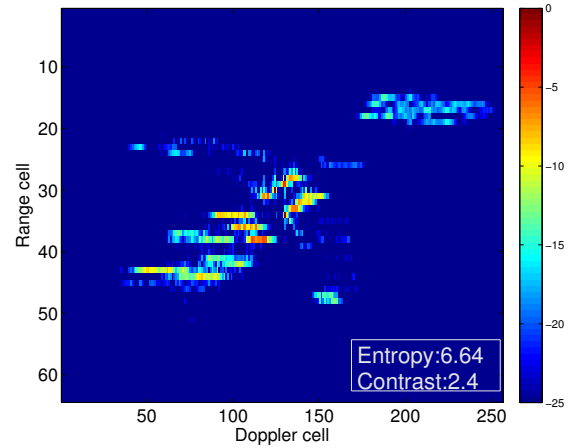

(b)

Figure 8. HRRPs and RD image. (a) HRRPs. (b) ISAR image based on RD algorithm.

To overcome the blurring, adaptive MFT is carried out and its imaging result is shown in Figure 9a; the corresponding RCR is 107.13. Compared with the result of RD in Figure 8b, adaptive MFT can considerably eliminate the blurring of the ISAR image by compensating the quadratic phase terms. A smaller entropy and larger contrast also validate the improvement in image quality. Next, we consider the residual translational compensation errors and take DFS into consideration. Simulation results show that the RCR and DFS are 106.57 and -0.52 , respectively. The imaging result is given in Figure $9 \mathrm{~b}$, which achieves the same image quality as Figure 9a, as described by the equal entropy and contrast. Due to the shift of Doppler focus being less than a Doppler cell, its effect on ISAR imaging can be neglected. The imaging result also indicates that linear residual translational compensation phase error does not exist in this data set.

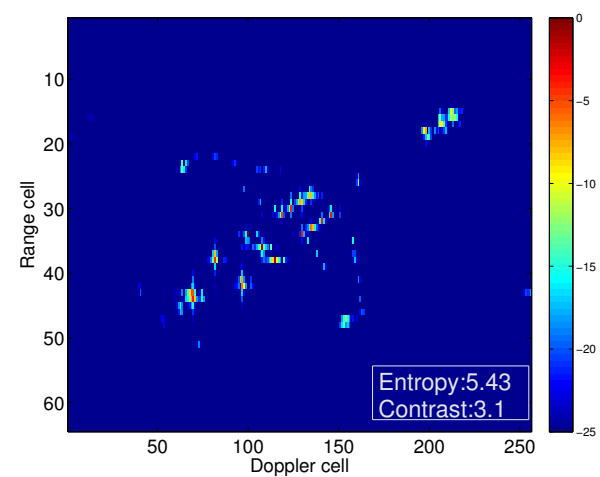

(a)

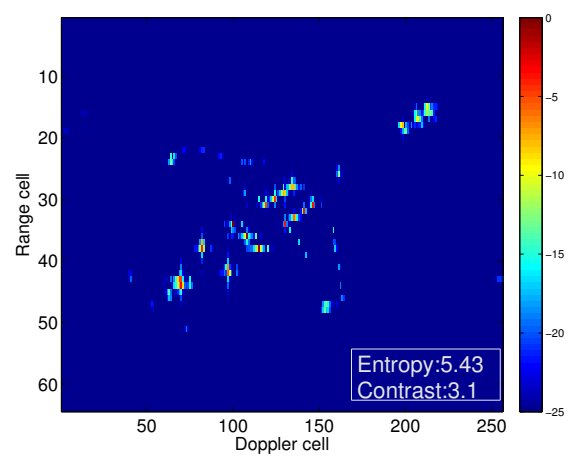

(b)

Figure 9. ISAR images of Boeing B727 aircraft. (a) ISAR image based on adaptive MFT. (b) ISAR image based on adaptive MFT with DFS compensation.

We next applied the new approach to obtain the ISAR image. Figure 10a shows the image entropy versus the iteration times during the iteration. As in the former experiment, the initial value of image entropy was set as the one of the RD images. It can be seen that from Figure 10a that after two iterations, the termination condition was met. The corresponding RCRs of the two iterations are 107.13 and 0 , respectively. Figure $10 \mathrm{~b}$ is the optimal imaging result by the new approach, from which we can see that the cross-range resolution was improved and some scatterers were more highly concentrated, especially scatterers located in the nose and wing of the airplane. It can be inferred that the non-linear residual translational compensation errors exist in the data set. Furthermore, we define them as non-linear phase errors. The image entropy of Figure $10 \mathrm{~b}$ is smallest of the four methods, and the contrast is the largest. Both image entropy and contrast confirm that the new imaging approach is more effective than other three methods. 


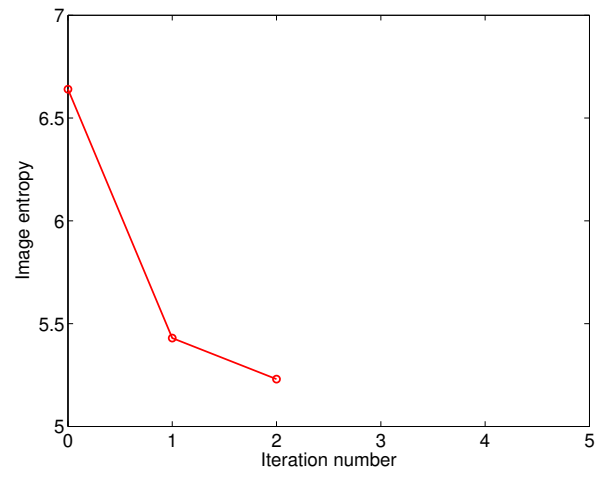

(a)

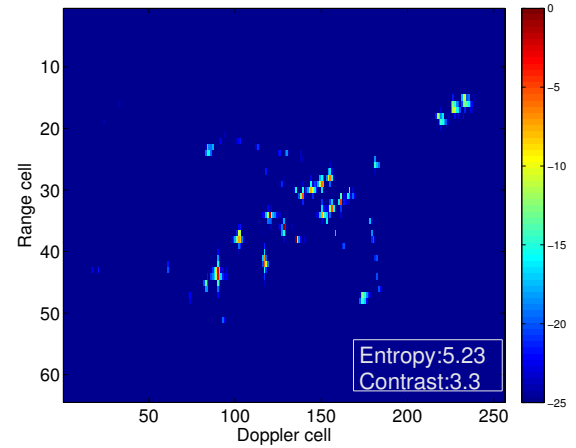

(b)

Figure 10. Experimental results using the new imaging approach. (a) Variation of the image entropy. (b) ISAR image based on the new imaging approach.

The above experimental results have validated the effectiveness of the new imaging approach based on iterative phase autofocus. The phase autofocus method for targets with complex motion will be researched in future work.

\section{Conclusions}

In this paper, a novel imaging approach based on iterative phase autofocus is proposed to achieve the translational phase autofocus for ISAR imaging of maneuvering targets. In the new approach, translational phase errors and adaptive MFT matrix are estimated iteratively. Ultimately, the phase autofocus of maneuvering targets converts to the phase autofocus of smooth-moving targets. Compared with precedent autofocus algorithms, the proposed approach combines phase autofocus and azimuth compression to obtain a global optimal ISAR image. Moreover, the new approach unifies the phase autofocus of maneuvering targets and smooth targets into the same model, which is useful and convenient for practical ISAR imaging system. Experimental results using simulated data and Boeing B727 data have validated the effectiveness and advantages of the new approach. Our future work will focus on the phase autofocus method for targets with complex motion.

Author Contributions: B.W.; H.C. proposed the method and wrote the manuscript; Z.Z.; H.T.; L.S.; B.D.; L.Z. revised the manuscript. All authors have read and agreed to the published version of the manuscript.

Funding: This work was supported by the China Postdoctoral Science Foundation (grant no. 2016M603007).

Data Availability Statement: All data included in this study are available upon request by contact with the corresponding author.

Conflicts of Interest: The authors declare no conflict of interest.

\section{References}

1. Kang, H.; Li, J.; Zhao, H.; Bao, Z.; Yu, Z. Calibration of Linear Time-Varying Frequency Errors for Distributed ISAR Imaging Based on the Entropy Minimization Principle. Sensors 2019, 19, 1323. [CrossRef] [PubMed]

2. Lazarov, A.; Minchev, C. ISAR Geometry, Signal Model and Image Processing Algorithms. IET Radar Sonar Navig. 2017, 11, 1425-1434. [CrossRef]

3. Zheng, J.; Liu, H.; Liu, Z.; Liu, Q. H. ISAR Imaging of Ship Targets Based on an Integrated Cubic Phase Bilinear Autocorrelation Function. Sensors 2018, 18, 498. [CrossRef] [PubMed]

4. Chen, C.C.; Andrews, H.C. Target-motion-induced radar imaging. IEEE Trans. Aerosp. Electron. Syst. 1980, 16, 2-14. [CrossRef]

5. Wang, Y.; Abdelkader, A.C.; Zhao, B.; Wang, J. ISAR Imaging of Maneuvering Targets Based on the Modified Discrete PolynomialPhase Transform. Sensors 2015, 15, 22401-22418. [CrossRef]

6. Wang, Y. Radar Imaging of Non-Uniformly Rotating Targets via a Novel Approach for Multi-Component AM-FM Signal Parameter Estimation. Sensors 2015, 15, 6905-6923. [CrossRef] 
7. Xing, M.D.; Bao, Z. Translational motion compensation and instantaneous imaging of ISAR maneuvering targets. Acta Electron. Sin. 2001, 29, 68-73. [CrossRef] [PubMed]

8. Liu, L.; Zhou, F.; Tao, M.L.; Sun, P.G.; Zhang, Z.J. Adaptive translational motion compensation method for ISAR imaging under low SNR based on particle swarm potimization. IEEE J. Sel. Top. Appl. Earth Obs. Remote. Sens. 2017, 8, 5146-5157. [CrossRef] [PubMed]

9. Liu, Y.; Hou, Q.K.; Xu, S.Y.; Tian, B. System distortion analysis and compensation of DIFS signals for wideband imaging radar. Sci. China Inf. Sci. 2015, 58, 1-16.

10. Zhu, Z.D.; Qiu, X.H.; She, Z.S. ISAR motion compensation using modified Doppler centroid tracking method. Acta Electron. Sin. 1997, 1, 359-363. [CrossRef]

11. Jakowatz, C.V.; Wahl, D.E.; Eichel, P.H.; Ghiglia, D.C.; Thompson, P.A. Phase Errors and Autofocus in SAR Imagery; Springer: Boston, MA, USA, 1996; pp. 221-271. [CrossRef]

12. Chan, H.L.; Yeo, T.S. Noniterative quality phase-gradient autofocus (QPGA) algorithm for spotlight SAR imagery. IEEE Trans. Geosci. Remote Sens. 1998, 36, 1531-1539.

13. Qiu, X.H.; Alice, H.W.C.; Yeo, S.Y. Fast minimum entropy phase compensation for ISAR imaging. J. Electron. Inf. Technol. 2004, 26, $1656-1660$.

14. Li, X.; Liu, G.S.; Ni, J.L. Autofocusing of ISAR images based on entropy minimization. IEEE Trans. Aerosp. Electron. Syst. 2002, 35, 1240-1252. [CrossRef]

15. Wang, B.; Xu, S.; Wu, W.; Hu, P.; Cheng, Z. Adaptive ISAR Imaging of Maneuvering Targets Based on a Modified Fourier Transform. Sensors 2018, 18, 1370.

16. Wang, J.F.; Kasilingam, D. Global range alignment for ISAR. IEEE Trans. Aerosp. Electron. Syst. 2003, 39, 351-357. [CrossRef]

17. Wu, W.Z.; Hu, P.J.; Xu, S.Y.; Chen, A.Z.; Chen, A.J. Image registration for InISAR based on joint translational motion compensation. IET Radar Sonar Navig. 2017, 11, 1597-1603. [CrossRef] [PubMed]

18. Li, D.; Liu, H.Q.; Gui, X.G.; Zhang, X.Z. An Efficient ISAR Imaging Method for Maneuvering Target Based on Synchrosqueezing Transform. IEEE Antennas Wirel. Propag. Lett. 2016, 15, 1317-1320. [CrossRef] 